\title{
Efecto de variables de paisaje y de rodal sobre la diversidad de especies arbóreas en el sotobosque de plantaciones de Pinus taeda en la provincia de Misiones, Argentina
}

\author{
Effect of landscape and stand variables on tree species diversity in the understory of Pinus taeda \\ plantations in Misiones province
}

\author{
Claudio J Dummel a*, Martin A Pinazo ${ }^{\text {b }}$ \\ *Autor de correspondencia: ${ }^{\text {a }}$ Universidad Nacional de Misiones, Facultad de Ciencias Forestales, \\ Bertoni 124, 3380 Eldorado, Argentina, cdummel@yahoo.com.ar \\ b Instituto Nacional de Tecnología Agropecuaria, EEA Montecarlo, Av. Libertador 2472, 3384, Montecarlo, Argentina, \\ mpinazo@montecarlo.inta.gov.ar
}

\begin{abstract}
SUMMARY
Forest plantations are within the most important productive activities in Misiones, Argentina. Afforestations are strongly criticized under the idea that are green deserts, non suitable as habitat for native fauna. The aim of the present study was to evaluate the regeneration of native tree species in the understory of Pinus taeda plantations and the influence of stand and landscape variables in the northwest of Misiones, Argentina. Plantations presenting different ages, previous land uses and landscape contexts were sampled. A total of 91 native tree species were found in the forest understory, and the most abundant were animal-dispersed tree species. The native tree composition of plantations understory was different from that of native forest. The similarity analysis revealed that old plantations were more similar to native forest than to young plantations. The canonical correspondence analysis (CCA) showed that previous land use was the variable with more influence on the understory composition, followed by the basal area and the distance to the native forest at the time of planting. Wind-dispersed and pioneer species were more associated with short distances to the native forest and lower basal area; whereas animal-dispersed and shade tolerant species were related to high basal area and previous land use. Results support the idea that forest plantations may represent a suitable environment for the development of native plants.
\end{abstract}

Key words: afforestation, understory, species richness, landscape.

\section{RESUMEN}

La producción forestal es una de las actividades más importante de la provincia de Misiones, Argentina. Estos cultivos son fuertemente criticados por la percepción de representar desiertos verdes, no aptos como hábitat para el desarrollo de especies nativas. El objetivo del presente trabajo fue evaluar la regeneración de especies arbóreas nativas en el sotobosque de forestaciones de Pinus taeda en el noroeste de la provincia de Misiones, considerando variables de rodal y de paisaje. Se muestrearon forestaciones de diferentes edades, usos anteriores del suelo y contextos de paisaje. Se identificaron 91 especies arbóreas nativas presentes en el sotobosque de las forestaciones, siendo más abundantes las dispersadas por animales. La composición del sotobosque de las plantaciones resultó diferente a la del bosque nativo. El análisis de similitud reveló que las forestaciones adultas resultaron más similares al bosque nativo que a las forestaciones jóvenes. El análisis de correspondencia canónico mostró al uso anterior del suelo como la variable que más influyó sobre la composición de las especies, seguido por el área basal de las forestaciones y la distancia al bosque nativo en el año de la plantación como variable de paisaje. La presencia de especies dispersadas por viento se asoció con la distancia al bosque nativo y baja área basal, mientras que la presencia de especies tolerantes lo hizo con alta área basal y con el uso anterior del suelo. Los resultados del presente trabajo refuerzan la consideración de las forestaciones como ambientes aptos para el desarrollo de la vegetación nativa.

Palabras clave: forestaciones, riqueza de especies, regeneración, selva paranaense.

\section{INTRODUCCIÓN}

La ecorregión del Bosque Atlántico del Alto Paraná es una de las ecorregiones más biodiversas del mundo y está incluida dentro de los ecosistemas más vulnerables según la iniciativa Global 200 del Fondo Mundial para la Naturaleza (WWF) (Olson y Dinerstein 2002). Actualmente solo el 7,8 \% de la cobertura original permanece como remanente de una región de 47.100 .000 ha a consecuencia de la deforestación para la habilitación de tierras para la realización de actividades agrícolas, ganaderas y forestales (Di Bitetti et al. 2003). La provincia de Misiones mantiene hasta el presente los remantes de mayor superficie de la ecorregión y a su vez concentra el 45 \% de la actividad forestal del país, mientras que el sector forestal representa el $50 \%$ del producto bruto de la provincia (SAGPyA 2001).

En los últimos años ha crecido la atención de la sociedad sobre el desarrollo de actividades productivas en el 
marco del uso sustentable del recurso y la conservación de la biodiversidad es uno de los temas que ha generado la preocupación de diferentes sectores de la sociedad a nivel mundial (Brockerhoff et al. 2008). En el caso particular de las forestaciones con especies exóticas de rápido crecimiento existe la percepción de "desierto verde" por parte de la sociedad (Brockerhoff et al. 2008). Sin embargo, diferentes autores mencionan a las forestaciones como hábitats aptos para animales, plantas, insectos entre otros seres vivos (Parrotta et al. 1997a). Es por ello que representarían una herramienta importante en la restauración de ambientes boscosos degradados (Lugo 1997, Parrotta et al. 1997a). Características propias de las forestaciones tales como especie, edad, cobertura del dosel y uso previo del suelo serían algunos de los factores que podrían influir sobre la diversidad y composición de especies del sotobosque (Brockerhoff et al. 2008), generando condiciones ambientales aptas para el arribo de propágulos e instalación y desarrollo de la regeneración de especies nativas (Lugo 1997). El desarrollo del dosel de la plantación modifica el régimen de radiación, temperatura, humedad, mejora los contenidos de nutrientes y materia orgánica de los suelos y restablece el ciclo de nutrientes (Lugo 1992, 1997). El proceso de colonización del sotobosque implica la llegada de propágulos y la existencia de condiciones aptas para la regeneración y desarrollo de las diferentes especies (Lugo 1997). Otros mecanismos de persistencia como el rebrote de raíz o el banco de semillas permitirían la presencia de especies en el sotobosque (Gachet et al. 2007). Sin embargo, estos mecanismos son fuertemente influenciados por el uso previo del suelo al eliminar el banco de propágulos y generar barreras que limitan la instalación de las especies (Holz et al. 2009).

A nivel de paisaje productivo se verifican procesos de pérdida de hábitat como consecuencia del cambio de uso del suelo y procesos de fragmentación de la vegetación natural. Estos procesos generan cambios en los patrones de hábitat y mosaicos de usos antrópicos del suelo combinados con remanentes de la vegetación natural (Turner et al. 2001). En este contexto, los tipos o elementos del paisaje, su arreglo espacial, el grado de fragmentación, sus proporciones, formas y conectividad influirían sobre la diversidad al generar ambientes que pueden ser aprovechados de manera diferencial por las especies (Lindenmayer y Hobbs 2004). La combinación de esquemas de manejo apropiados a nivel de rodal y de paisaje de las forestaciones puede generar ambientes importantes para el mantenimiento de la biodiversidad a nivel de paisaje (Hartley 2002, Deconchat $e t$ al. 2009). Por lo expuesto, en el presente trabajo se plantea la hipótesis que las forestaciones de Pinus taeda L. constituyen un ambiente apto para la instalación de especies arbóreas nativas en el sotobosque y que la composición de especies es influenciada por variables de paisaje, características de desarrollo propias de los rodales y la historia de uso del sitio. El objetivo del presente estudio es explorar la influencia de variables de paisaje y de rodal sobre la diversidad de especies leñosas en el sotobosque de plantaciones de Pinus taeda en el norte de la provincia de Misiones, Argentina.

\section{MÉTODOS}

El muestreo se llevó a cabo en el sector noroeste de la provincia de Misiones, Argentina, área en que dominan las forestaciones de Pinus taeda por sobre las plantaciones de Pinus elliottii Engelm. y Eucalyptus grandis W. Hill ex Maiden. La región corresponde fitogeograficamente a la provincia paranaense, distrito de las selvas mixtas (Cabrera 1976). El bosque original es multiestratificado con tres estratos arbóreos, un estrato de bambúceas y arbustos, estrato herbáceo y estrato muscinal. Además, hay uno o más estratos de epifitas y un estrato de lianas (Cabrera 1976). El clima de la región se caracteriza por temperaturas medias anuales de $21^{\circ} \mathrm{C}$ y un régimen de precipitaciones isohigro con valores de $2.000 \mathrm{~mm}$ anuales. Los suelos son lateríticos, derivados de rocas basálticas y corresponden a los Kandiudultes, desarrollados sobre una topografía plana a ondulada.

Se muestrearon 19 plantaciones forestales de Pinus taeda de diferentes edades y en diferentes contextos de paisaje (figura 1). Los parches de forestación presentaron superficies que variaron de 6 a 160 hectáreas, con un valor promedio de 25,7 hectáreas. En líneas generales, la densidad de plantación es de 1.100 a 1.600 árboles ha-1. La preparación del suelo se realiza mediante la utilización de arado o subsolador y control de malezas mediante métodos químicos hasta el tercer año. Si bien los regímenes silvícolas pueden variar entre los propietarios de las plantaciones muestreadas, estas estarían bajo un sistema intensivo ${ }^{1}$. Este sistema implica la realización de raleos periódicos y podas hasta una altura de 5,5 metros. Adicionalmente, y con el objeto de contrastar la composición de especies, se realizaron muestreos en cuatro sitios de bosque nativo secundario remanente dentro de tierras con uso agrícola y forestal, cercanos a las forestaciones muestreadas. Los remanentes presentaban diferentes grados de uso y los tamaños oscilaron entre 21 a 270 hectáreas. En cada sitio de forestación y bosque nativo se establecieron parcelas circulares concéntricas anidadas modificado a partir del diseño del Programa de Monitoreo de Sanidad Forestal, Departamento de Agricultura de los Estados Unidos. Este diseño es utilizado para el monitoreo de la diversidad de plantas a diferentes escalas conjuntamente con otras variables de rodal (Stohlgren 2007). Las parcelas se ubicaron en los vértices de un cuadrado de $100 \mathrm{~m}$ de lado a más de $50 \mathrm{~m}$ del borde del límite del ambiente a muestrear para evitar el efecto de borde. En parcelas de $400 \mathrm{~m}^{2}$ se midieron las variables diámetro a la altura del pecho (DAP), densidad y área basal del rodal. En parcelas de $100 \mathrm{~m}^{2}$ se determinaron las especies arbóreas mayores a 1,3 m de altura presentes en

\footnotetext{
${ }^{1}$ Hugo Fassola. INTA EEA Montecarlo, Misiones, Argentina. Comunicación personal.
} 
el sotobosque y en parcelas de $25 \mathrm{~m}^{2}$ las especies arbóreas mayores a $0,3 \mathrm{~m}$ y menores a $1,3 \mathrm{~m}$ de altura. Todos los individuos presentes fueron determinados a nivel de especie. Posteriormente, las especies fueron clasificadas de acuerdo al tipo de dispersión de sus semillas y los requerimientos de luz (Carvalho 2003). Los sitios fueron agrupados en plantaciones jóvenes (edades hasta 15 años), plantaciones adultas (15 a 30 años de edad) y bosque nativo (cuadro 1).

En cada punto de muestreo se definieron tres escalas de análisis del paisaje a partir de círculos de 500 - 1.000 y $2.000 \mathrm{~m}$ de radio. La máxima distancia de análisis se determinó buscando evitar la auto correlación de los datos debido la superposición de las áreas definidas por el radio de 2.000 m y en función a las distancias de dispersión por parte de diferentes agentes y a la influencia del paisaje sobre la abundancia de aves informados en otras regiones (Deconchat et al. 2009). Para cada círculo se digitalizaron visualmente, a partir de imágenes satelitales Landsat MMS

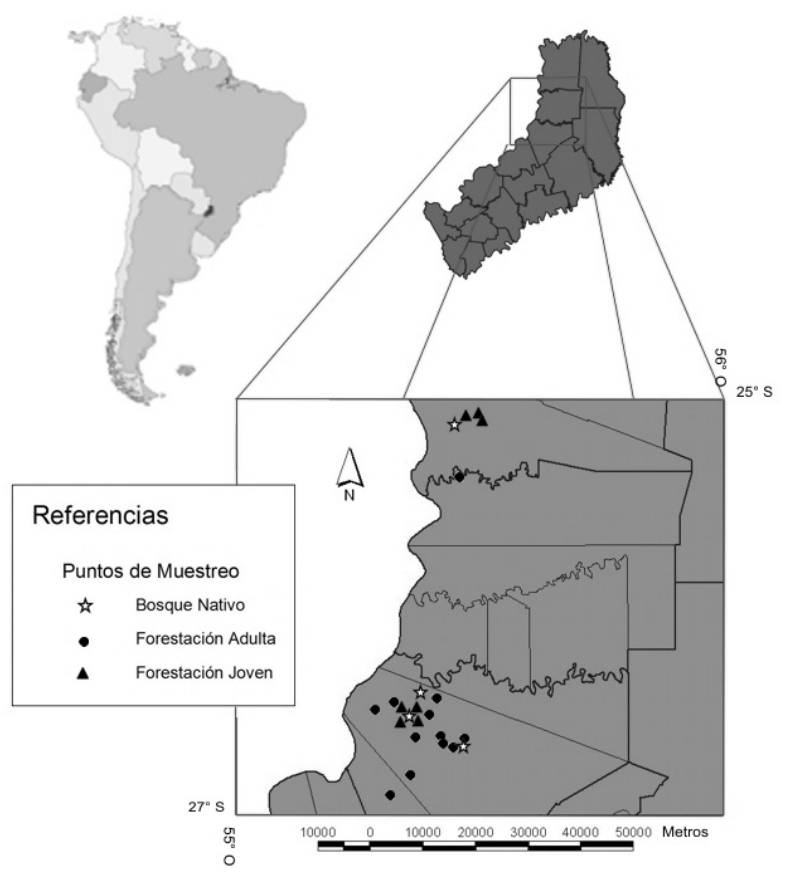

Figura 1. Ubicación de los sitios de muestreo. Location of sample sites. y Landsat-V TM y mediante un sistema de información geográfica, los usos de la tierra desde el punto de muestreo para las categorías: 1) bosque nativo, 2) bosque secundario, 3) plantación y 4) agricultura. Como las especies presentes en el sotobosque de las forestaciones pueden representar fuentes de semillas de parches de bosque no presentes en la actualidad, se estimaron las métricas de paisaje y los usos de la tierra para cada escala de análisis para los años 1973, 1989 y 2003. Las métricas de paisaje estimadas fueron: distancia desde el punto de muestreo al borde de bosque nativo más cercano, porcentaje de clase de uso de la tierra y tamaño de la forestación para las tres escalas y años mencionados. Las imágenes utilizadas fueron MSS Landsat para el año 1973 (imágenes 240 - 79 y 240 - 78), Landsat TM IV para el año 1989 y Landsat-V TM para el año 2003 (imagen 224 - 78). Las imágenes fueron analizadas mediante ARCGIS 9.0 y las métricas de paisaje fueron estimadas utilizando Fragstat 3.3 (McGarigal et al. 2002).

Análisis de los datos. Se analizó el esfuerzo de muestreo necesario mediante los índices no paramétricos Chao 2 y Bootstrap. Para comparar la riqueza promedio de especies y la diversidad (mediante el índice de Shannon) en los diferentes grupos o tratamientos (plantaciones jóvenes, plantaciones adultas y bosque nativo), se aplicó el análisis de la varianza (ANDEVA) de una vía, y se compararon las medias mediante la prueba de Tukey.

Para comparar las similitudes en la composición de especies entre tipos de ambientes se realizó un análisis de similitud mediante el índice de Bray-Curtis (Clarke 1993) con 5.000 réplicas utilizando el programa PAST 1.82 (Hammer et al. 2001). La prueba utiliza el estadístico R el cual puede tomar valores entre 0 y 1 , representando los valores positivos cercanos a uno disimilitud entre grupos.

Para explorar la influencia del paisaje y las variables estructurales del rodal sobre la composición del sotobosque en las plantaciones se realizó un análisis de correspondencia canónica (Ter Braak y Šmilauer 1998). Se utilizaron las métricas de paisaje para cada escala de análisis y año analizado, y las variables estructurales de rodal (área basal y densidad) como variables ambientales o independientes, representando un total de 48 variables. Como variable respuesta o dependiente se utilizó la abundancia de las especies. Debido a la gran cantidad de variables am-

Cuadro 1. Características estructurales de los grupos de forestaciones jóvenes, forestaciones adultas y bosque nativo. Valores promedio y desvío estándar.

Structural parameter of groups of young plantations, old plantations and native forest. Mean values and standard deviation.

\begin{tabular}{lcccc}
\hline Tipos de vegetación & Sitios Muestreados & $\begin{array}{c}\text { DAP } \\
\text { medio }(\mathrm{cm})\end{array}$ & $\begin{array}{c}\text { Área basal } \\
\left(\mathrm{m}^{2} \text { ha }^{-1}\right)\end{array}$ & $\begin{array}{c}\text { Densidad } \\
(\text { individuos ha }\end{array}$ \\
\hline Bosque nativo & 4 & $24,55 \pm 1,10$ & $16,37 \pm 3,88$ & $247 \pm 45$ \\
Forestación adulta & 12 & $37,74 \pm 4,58$ & $25,03 \pm 5,35$ & $232 \pm 97$ \\
Forestación joven & 7 & $22,34 \pm 6,73$ & $20,47 \pm 3,94$ & $597 \pm 260$ \\
\hline
\end{tabular}


bientales, se seleccionaron aquellas variables no correlacionadas entre sí, a través de la matriz de correlación (Ter Braak y Šmilauer 1998). Posteriormente, se incluyeron en el análisis sólo las variables que presentaban significancia de probabilidad menor a 0,05 para explicar la variabilidad de los datos. Se utilizó la opción de bajar la influencia de las especies raras para evitar la distorsión de los resultados (Ter Braak y Šmilauer 1998). Para evaluar la significación estadística de los diferentes ejes del análisis de correspondencia canónica, se utilizó la prueba de permutaciones de Monte Carlo (Ter Braak y Šmilauer 1998). Cada eje fue evaluado a un nivel de $95 \%$ de confianza, con 500 permutaciones. El análisis se realizó mediante el software CANOCO 4.5 (Ter Braak y Šmilauer 1998).

\section{RESULTADOS}

Diversidad de especies. En total se registraron 48 especies arbóreas en las forestaciones jóvenes, 88 especies en las forestaciones adultas y 79 especies en los remanentes de bosque nativo. Sólo se registraron especies exóticas en las forestaciones, con dos especies en las jóvenes y ocho especies en las adultas (cuadro 2). Los índices no paramétricos utilizados para evaluar el esfuerzo de muestreo arrojaron valores satisfactorios (cuadro 2). Los valores de especies inventariadas representaron valores de $79 \%$ a $90 \%$ respecto a las estimadas mediante Chao 2. En el caso de Bootstrap, los valores resultaron más próximos al número de especies encontradas con porcentajes de $97 \%$ para forestaciones adultas y valores porcentuales de $88 \%$ y $90 \%$ para las forestaciones jóvenes y bosque nativo respectivamente.

En el total de los sitios de forestaciones muestreados se encontraron 91 especies arbóreas regenerando en el sotobosque de las mismas (cuadro 3), correspondiendo a 38 familias y 83 géneros. Las familias más abundantes fueron Fabaceae (13 géneros y 18 especies) representando el 16,7 \% del total de las especies, seguida por las Myrtaceae (cuatro géneros y nueve especies) con 8,3 \% y Rutaceae (cinco géneros y siete especies) con $6,48 \%$.

Los valores promedio del número de especies inventariadas resultaron ser significativamente distintos $(\mathrm{F}=28,45$;
$P<0,001)$ entre los tres grupos, con un gradiente decreciente bosque nativo, forestaciones adultas y forestaciones jóvenes (figura 2). Por su parte, el índice de Shannon mostró la misma tendencia resultando las diferencias en los valores del índice significativas entre los tres tratamientos ( $\mathrm{F}=14,29 ; P<0,001$ ) (cuadro 2).

El análisis de similitud mostró que la composición de especies en las forestaciones resultó significativamente diferente respecto al bosque nativo. Las forestaciones adultas y jóvenes presentaron valores del índice de $0,43(P<0,006)$ y $0,77(P<0,003)$ respecto al bosque nativo. Las forestaciones adultas, al presentar un valor del índice menor, mostraron una disimilitud menor con el bosque nativo a diferencia de las forestaciones jóvenes que presentaron la mayor disimilitud. Por otra parte las forestaciones jóvenes y adultas presentaron un valor de 0,44 $(P<0,001)$ entre sí indicando composiciones relativamente similares.

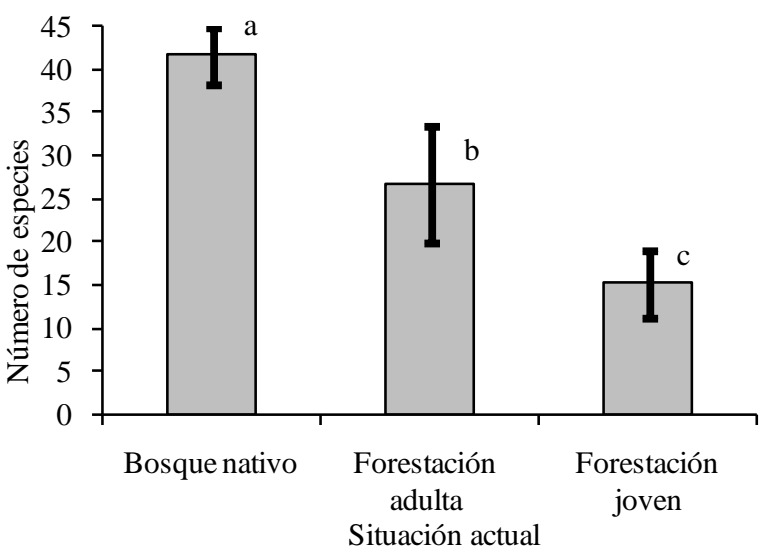

Figura 2. Riqueza de especies para forestaciones jóvenes, adultas y bosque nativo. Las columnas representan los valores promedio de los tratamientos. Las barras representan el error típico. Letras diferentes indican diferencias significativas (Tukey, $P<0,01$ ).

Species richness in young plantations, old plantations and native forest. Columns represent mean values and the bars represent the standard error. Different letters indicate significant differences (Tukey, $P<0.01)$.

Cuadro 2. Riqueza de especies, estimadores de esfuerzo de muestreo, e índice de diversidad de Shannon (H) para los grupos de forestaciones jóvenes, forestaciones adultas y bosque nativo. Letras diferentes indican diferencias significativas (Tukey, $P<0,01$ ).

Species richness, non parametrical estimator of richness and Shannon diversity index $(\mathrm{H})$ in the different groups of young plantations, old plantation and native forest. Different letters indicate significant differences (Tukey, $P<0.01$ ).

\begin{tabular}{|c|c|c|c|c|c|c|c|}
\hline \multirow{2}{*}{ Tipo de vegetación } & \multicolumn{2}{|c|}{ Total de especies } & \multicolumn{4}{|c|}{ Representatividad del muestreo } & \multirow{2}{*}{$\begin{array}{c}\text { Diversidad } \\
\text { Shannon }(\mathrm{H})\end{array}$} \\
\hline & Nativas & Exóticas & Chao 2 & $\%$ muestreo & Bootstrap & $\%$ muestreo & \\
\hline Bosque nativo & 79 & 0 & 90,52 & 87,3 & 87,86 & 89,9 & 2,99a \\
\hline Forestación adulta & 80 & 8 & 98,13 & 89,7 & 89,96 & 97,8 & $2,77 b$ \\
\hline Forestación joven & 46 & 2 & 61,05 & 78,6 & 54,42 & 88,2 & $2,06 \mathrm{c}$ \\
\hline
\end{tabular}


Cuadro 3. Listado de las especies identificadas en los grupos de forestaciones. ${ }^{*}$ Observed species in the plantation groups.

\begin{tabular}{|c|c|c|c|c|c|c|}
\hline Especie & Código & Familia & TD & Presencia & RL & GE \\
\hline Acacia nitidifolia Speg. & ACANIT & Fabaceae & $\mathrm{O}$ & $\mathrm{BN}$ & - & P-SI \\
\hline Acacia recurva Benth. & ACAREC & Fabaceae & $\mathrm{O}$ & FA-FJ-BN & - & P-SI \\
\hline Acacia tucumanensis Griseb. & ACATUC & Fabaceae & $\mathrm{O}$ & FA-FJ-BN & - & P-SI \\
\hline $\begin{array}{l}\text { Achatocarpus praecox Griseb. f. obovatus } \\
\text { (Schinz et Autran) Hauman }\end{array}$ & ACHPRA & Achatocarpaceae & $\mathrm{Z}$ & $\mathrm{BN}$ & - & SI-ST \\
\hline Acnistus arborescens (L.) Schltdl. & ACNARB & Solanaceae & $\mathrm{Z}$ & $\mathrm{BN}$ & - & - \\
\hline Actinostemon concolor (Spreng.) Müll. Arg. & ACTCON & Euphorbiaceae & $\mathrm{O}$ & FA-BN & - & ST \\
\hline Aegiphila brachiata Briq. Vell. & AEGHAS & Verbenaceae & $\mathrm{Z}$ & FA-FJ & $\mathrm{H}$ & P-SI \\
\hline Albizia niopoides (Spruce ex Benth.) Burkart & ALBNIO & Fabaceae & $\mathrm{O}$ & FA-FJ-BN & $\mathrm{H}$ & P-SI \\
\hline $\begin{array}{l}\text { Alchornea glandulosa Poepp. subsp. iricurana } \\
\text { (Casar.) Secco }\end{array}$ & ALCGLA & Euphorbiaceae & Z & FA-FJ & $\mathrm{H}$ & SI \\
\hline${ }^{\dagger}$ Aleurites fordii Hemsl. & ALEFOR & Euphorbiaceae & $\mathrm{O}$ & FA & - & - \\
\hline $\begin{array}{l}\text { Allophylus edulis (A. St.-Hil., A. Juss. et } \\
\text { Cambess.) Hieron. ex Niederl. }\end{array}$ & ALLEDU & Sapindaceae & $\mathrm{Z}$ & FA-FJ-BN & E & SI-ST \\
\hline Allophylus guaraniticus (A. St.-Hil.) Radlk. & ALLGUA & Sapindaceae & Z & FA & - & - \\
\hline Aloysia virgata (Ruiz et Pav.) Juss. var. virgata & ALOVIR & Verbenaceae & $\mathrm{O}$ & FA-FJ & $\mathrm{H}$ & P-SI \\
\hline Apuleia leiocarpa (Vogel) J.F. Macbr. & APULEI & Fabaceae & A & FA-FJ-BN & I & SI-ST \\
\hline $\begin{array}{l}\text { Araucaria angustifolia (Bertol.) Kuntze var. } \\
\text { angustifolia }\end{array}$ & ARAANG & Araucariaceae & $\mathrm{Z}$ & FA-FJ & I & SI-ST \\
\hline Aspidosperma australe Müll. Arg. & ASPAUS & Apocynaceae & A & FA-FJ-BN & - & ST-C \\
\hline Balfourodendron riedelianum (Engl.) Engl. & BALRIE & Rutaceae & A & FA-BN & I & ST \\
\hline Bastardiopsis densiflora (Hook. et Arn.) Hassl. & BASDEN & Malvaceae & $\mathrm{O}$ & FA-BN & $\mathrm{H}$ & P-SI \\
\hline $\begin{array}{l}\text { Bauhinia forficata Link subsp. pruinosa (Vogel) } \\
\text { Fortunato et Wunderlin }\end{array}$ & BAUFOR & Fabaceae & $\mathrm{O}$ & FA-FJ-BN & $\mathrm{H}$ & P-SI \\
\hline Cabralea canjerana (Vell.) Mart. & CABCAN & Meliaceae & $\mathrm{Z}$ & FA-FJ-BN & E & SI-ST \\
\hline Calliandra foliolosa Benth. & CALFOL & Fabaceae & $\mathrm{O}$ & FA-FJ-BN & - & ST-C \\
\hline $\begin{array}{l}\text { Campomanesia guazumifolia (Cambess.) O. } \\
\text { Berg }\end{array}$ & CAMGUA & Myrtaceae & $\mathrm{Z}$ & $\mathrm{BN}$ & I & ST \\
\hline $\begin{array}{l}\text { Campomanesia xanthocarpa O. Berg var. } \\
\text { xanthocarpa }\end{array}$ & CAMXAN & Myrtaceae & $\mathrm{Z}$ & FA-BN & E & ST \\
\hline${ }^{\dagger}$ Carica papaya L. & CARPAP & Caricaceae & Z & FA & - & - \\
\hline Casearia decandra Jacq. & CASDEC & Flacourtiaceae & $\mathrm{Z}$ & $\mathrm{BN}$ & E & ST \\
\hline Casearia sylvestris Sw. var. sylvestris & CASSYL & Flacourtiaceae & Z & FJ & E & ST \\
\hline Cecropia pachystachya Trécul & СЕСРАC & Cecropiaceae & $\mathrm{Z}$ & FA-FJ-BN & $\mathrm{H}$ & $\mathrm{P}$ \\
\hline Cedrela fissilis Vell. & CEDFIS & Meliaceae & A & FA-FJ-BN & I & SI-ST \\
\hline $\begin{array}{l}\text { Ceiba speciosa (A. St.-Hil, Juss et Cambess) } \\
\text { Ravenna }\end{array}$ & CEISPE & Bombacaceae & A & FA-BN & $\mathrm{H}$ & SI-ST \\
\hline Celtis iguanaea (Jacq.) Sarg. & CELIGU & Celtidaceae & Z & FA-BN & - & P-SI \\
\hline Cestrum laevigatum Schltdl. & CESLAE & Solanaceae & Z & FA-FJ-BN & - & - \\
\hline $\begin{array}{l}\text { Chrysophyllum gonocarpum (Mart. et Eichler) } \\
\text { Engl. }\end{array}$ & CHRGON & Sapotaceae & $\mathrm{Z}$ & FA-FJ-BN & E & ST \\
\hline $\begin{array}{l}\text { Chrysophyllum marginatum (Hook. et Arn.) } \\
\text { Radlk. subsp. marginatum }\end{array}$ & CHRMAR & Sapotaceae & Z & $\mathrm{BN}$ & - & ST \\
\hline
\end{tabular}




\begin{tabular}{|c|c|c|c|c|c|c|}
\hline${ }^{\dagger}$ Citrus sp. L. & CITSPP & Rutaceae & $\mathrm{Z}$ & FA-BN & - & - \\
\hline Cordia ecalyculata Vell. & CORECA & Boraginaceae & $\mathrm{Z}$ & FA-FJ-BN & I & SI-ST \\
\hline Cordia trichotoma (Vell.) Arráb. ex Steud. & CORTRI & Boraginaceae & A & FA-FJ-BN & I & SI-ST \\
\hline Cupania vernalis Cambess. & CUPVER & Sapindaceae & $\mathrm{Z}$ & FA-FJ-BN & $\mathrm{E}$ & ST \\
\hline Dalbergia frutescens (Vell.) Britton & DALFRU & Fabaceae & A & FA-FJ-BN & I & SI-ST \\
\hline Diatenopteryx sorbifolia Radlk. & DIASOR & Sapindaceae & A & FA-BN & - & ST \\
\hline${ }^{\dagger}$ Eriobotrya japonica (Thunb.) Lindl. & ERIJAP & Rosaceae & Z & FA & - & - \\
\hline $\begin{array}{l}\text { Erythroxylum deciduum A. St.-Hil. var. } \\
\text { decidum }\end{array}$ & ERYDEC & Erythroxylaceae & Z & FA & - & - \\
\hline Eugenia burkartiana (D. Legrand) D. Legrand & EUGBUR & Myrtaceae & Z & FA-BN & E & - \\
\hline Eugenia involucrata DC. & EUGINV & Myrtaceae & Z & $\mathrm{BN}$ & E & ST \\
\hline Eugenia pyriformis Cambess. var. pyriformis & EUGPIR & Myrtaceae & Z & FA-BN & $\mathrm{E}$ & - \\
\hline Eugenia ramboi D. Legrand & EUGRAM & Myrtaceae & Z & FA & $\mathrm{E}$ & - \\
\hline Eugenia uniflora $\mathrm{L}$. & EUGUNI & Myrtaceae & $\mathrm{Z}$ & FA & $\mathrm{E}$ & SI-ST \\
\hline Zanthoxylum fagara (L.) Sarg. & FAGHYE & Rutaceae & $\mathrm{O}$ & $\mathrm{FA}$ & I & ST \\
\hline Zanthoxylum petiolare A. St.-Hil. et Tul. & FARNAR & Rutaceae & $\mathrm{O}$ & $\mathrm{BN}$ & I & - \\
\hline Zanthoxylum rhoifolium Lam. & FAGRHO & Rutaceae & $\mathrm{O}$ & FA & I & P-SI \\
\hline Ficus luschnathiana (Miq.) Miq. & FICLUS & Moraceae & Z & FA-BN & $\mathrm{E}$ & SI-ST \\
\hline $\begin{array}{l}\text { Guarea macrophylla Vahl subsp. spicaeflora } \\
\text { (A. Juss.) T.D. Penn. }\end{array}$ & GUAMAC & Meliaceae & Z & $\mathrm{BN}$ & - & - \\
\hline Helietta apiculata Benth. & HELAPI & Rutaceae & A & FA & $\mathrm{H}$ & SI-ST \\
\hline Heliocarpus popayanensis Kunth & HELPOP & Tiliaceae & A & FA & $\mathrm{H}$ & $\mathrm{P}$ \\
\hline Hennecartia omphalandra J. Poiss. & HENOMP & Monimiaceae & Z & FA-BN & - & - \\
\hline Holocalyx balansae Micheli & HOLBAL & Fabaceae & $\mathrm{Z}$ & FA-BN & E & $\mathrm{C}$ \\
\hline${ }^{\dagger}$ Hovenia dulcis Thunb. & HOVDUL & Rhamnaceae & $\mathrm{Z}$ & $\mathrm{FA}$ & - & - \\
\hline $\begin{array}{l}\text { Ilex paraguariensis A. St.-Hil. var. } \\
\text { paraguariensis }\end{array}$ & ILEPAR & Aquifoliaceae & Z & FA-FJ-BN & $\mathrm{E}$ & $\mathrm{C}$ \\
\hline Inga marginata Willd. & INGSEM & Fabaceae & Z & FA-FJ-BN & $\mathrm{H}$ & SI \\
\hline Inga uraguensis Hook. et Arn. & INGVER & Fabaceae & Z & $\mathrm{BN}$ & $\mathrm{H}$ & $\mathrm{P}$ \\
\hline Jacaratia spinosa (Aubl.) A. DC. & JACSPI & Caricaceae & $\mathrm{Z}$ & $\mathrm{BN}$ & E & P-ST \\
\hline Lonchocarpus campestris Mart. ex Benth. & LONLEU & Fabaceae & A & FA-FJ-BN & - & ST \\
\hline Lonchocarpus muehlbergianus Hassl. & LONMUE & Fabaceae & A & FA-FJ-BN & $\mathrm{H}$ & SI-ST \\
\hline Luehea divaricata Mart. & LUEDIV & Tiliaceae & $\mathrm{O}$ & FA & I & SI-ST \\
\hline Machaerium stipitatum (DC.) Vogel & MACMIN & Fabaceae & A & FA-FJ-BN & I & ST \\
\hline Machaerium paraguariense Hassl. & MACPAR & Fabaceae & A & FA-FJ-BN & I & ST \\
\hline Maclura tinctoria (L.) Steud. subsp. tinctoria & MACTIN & Moraceae & Z & FA-FJ-BN & $\mathrm{H}$ & SI \\
\hline Manihot grahamii Hook. & MANGRA & Euphorbiaceae & Z & FA-FJ-BN & $\mathrm{H}$ & $\mathrm{P}$ \\
\hline Matayba elaeagnoides Radlk. & MATELE & Sapindaceae & $\mathrm{Z}$ & FA-FJ-BN & $\mathrm{E}$ & ST \\
\hline${ }^{\dagger}$ Melia azederach L. & MELAZE & Meliaceae & $\mathrm{Z}$ & FA-FJ & - & - \\
\hline${ }^{\dagger}$ Morus nigra L. & MORNIG & Moraceae & $\mathrm{Z}$ & FA-FJ & - & - \\
\hline Myrcia bombycina (O. Berg) Kiaersk. & MYRBOM & Myrtaceae & Z & FA & - & ST \\
\hline Myrocarpus frondosus Allemão & MYRFRO & Fabaceae & A & FA-FJ-BN & I & SI-ST \\
\hline
\end{tabular}




\begin{tabular}{|c|c|c|c|c|c|c|}
\hline Myrsine balansae (Mez) Otegui & MYRBAL & Myrsinaceae & $\mathrm{Z}$ & FA-BN & $\mathrm{H}$ & SI \\
\hline Myrsine coriacea (Sw.) R. Br. & MYRCOR & Myrsinaceae & $\mathrm{Z}$ & FA & $\mathrm{H}$ & SI \\
\hline Nectandra lanceolata Nees et Mart. ex Nees & NECLAN & Lauraceae & $\mathrm{Z}$ & FA-FJ-BN & I & ST \\
\hline Nectandra megapotamica (Spreng.) Mez & NECMEG & Lauraceae & $\mathrm{Z}$ & FA-FJ-BN & $\mathrm{E}$ & SI-ST \\
\hline Ocotea diospyrifolia (Meisn.) Mez & OCODIO & Lauraceae & $\mathrm{Z}$ & FA-BN & - & ST \\
\hline Ocotea puberula (Rich.) Nees & OCOPUB & Lauraceae & $\mathrm{Z}$ & FA-FJ-BN & I & SI \\
\hline Ocotea pulchella (Nees) Mez & OCOPUL & Lauraceae & $\mathrm{Z}$ & FA & $\mathrm{H}$ & ST \\
\hline Parapiptadenia rigida (Benth.) Brenan & PARRIG & Fabaceae & A & FA-FJ-BN & I & I-SI-ST \\
\hline Cordia americana (L.) Gottschling et J.S. Mill. & PATAME & Boraginaceae & A & FA-FJ-BN & I & SI-ST \\
\hline Peltophorum dubium (Spreng.) Taub. & PELDUB & Fabaceae & A & FJ-BN & $\mathrm{H}$ & SI \\
\hline Pereskia aculeata Mill. & PERACU & Cactaceae & $\mathrm{Z}$ & FA-BN & - & - \\
\hline Picrasma crenata (Vell.) Engl. & PICCRE & Simaroubaceae & $\mathrm{Z}$ & $\mathrm{BN}$ & - & - \\
\hline Pilocarpus pennatifolius Lem. & PILPEN & Rutaceae & $\mathrm{O}$ & FA-BN & - & - \\
\hline${ }^{\dagger}$ Pinus elliottii Engl. & PINELL & Pinaceae & A & FA & - & - \\
\hline Plinia rivularis (Cambess.) Rotman & PLIRIV & Myrtaceae & $\mathrm{Z}$ & FA & - & - \\
\hline $\begin{array}{l}\text { Prunus brasiliensis (Cham. et Schltdl.) D. } \\
\text { Dietr. }\end{array}$ & PRUBRA & Rosaceae & $\mathrm{Z}$ & FA-FJ-BN & $\mathrm{H}$ & SI \\
\hline Rollinia rugulosa Schltdl. & ROLRUG & Annonaceae & $\mathrm{Z}$ & FA & I & SI \\
\hline Rollinia salicifolia Schltdl. & ROLSAL & Annonaceae & $\mathrm{Z}$ & FA-FJ-BN & I & SI \\
\hline Ruprechtia laxiflora Meisn. & RUPLAX & Polygonaceae & A & FA-BN & $\mathrm{H}$ & ST \\
\hline Sapium glandulosum (L.) Morong & SAPGLA & Euphorbiaceae & $\mathrm{O}$ & FA & $\mathrm{H}$ & SI \\
\hline $\begin{array}{l}\text { Schinus terebinthifolius Raddi var. pohlianus } \\
\text { Engl. }\end{array}$ & SCHTER & Anacardiaceae & $\mathrm{Z}$ & FA-FJ & $\mathrm{H}$ & P-SI \\
\hline Sebastiania brasiliensis Spreng. & SEBBRA & Euphorbiaceae & $\mathrm{O}$ & $\mathrm{BN}$ & $\mathrm{H}$ & SI \\
\hline Seguieria aculeata Jacq. & SEGACU & Phytolaccaceae & A & $\mathrm{BN}$ & - & - \\
\hline Solanum granulosum-leprosum Dunal & SOLGRA & Solanaceae & $\mathrm{Z}$ & FA-FJ-BN & $\mathrm{H}$ & $\mathrm{P}$ \\
\hline Solanum inaequale Vell. & SOLINA & Solanaceae & $\mathrm{Z}$ & FA-FJ-BN & $\mathrm{H}$ & $\mathrm{P}$ \\
\hline Solanum ramulosum Sendtn. & SOLRAM & Solanaceae & $\mathrm{Z}$ & $\mathrm{BN}$ & $\mathrm{H}$ & $\mathrm{P}$ \\
\hline Solanum sanctae-catharinae Dunal & SOLSAN & Solanaceae & $\mathrm{Z}$ & $\mathrm{BN}$ & $\mathrm{H}$ & $\mathrm{P}$ \\
\hline $\begin{array}{l}\text { Sorocea bonplandii (Baill.) W.C. Burger, Lanj. } \\
\text { et Wess. Boer }\end{array}$ & SORBON & Moraceae & $\mathrm{Z}$ & FA-BN & $\mathrm{E}$ & $\mathrm{C}$ \\
\hline Strychnos brasiliensis (Spreng.) Mart. & STRYBRA & Loganiaceae & $\mathrm{Z}$ & FA-BN & - & - \\
\hline Styrax leprosus Hook. et Arn. & STYLEP & Styracaceae & $\mathrm{Z}$ & FA-BN & $\mathrm{E}$ & ST \\
\hline Syagrus romanzoffiana (Cham.) Glassman & SYAROM & Palmae & $\mathrm{Z}$ & FA-BN & $\mathrm{H}$ & SI \\
\hline Tabernaemontana catharinensis A. DC. & TABCAT & Apocynaceae & $\mathrm{Z}$ & FA-FJ & - & - \\
\hline Trema micrantha (L.) Blume & TREMIC & Celtidaceae & $\mathrm{Z}$ & FA-FJ-BN & $\mathrm{H}$ & $\mathrm{P}$ \\
\hline Trichilia catigua A. Juss. & TRICAT & Meliaceae & $\mathrm{Z}$ & FA-BN & $\mathrm{E}$ & ST-C \\
\hline Trichilia claussenii C. DC. & TRICLA & Meliaceae & $\mathrm{Z}$ & $\mathrm{BN}$ & $\mathrm{E}$ & ST-C \\
\hline Vitex megapotamica (Spreng.) Moldenke & VITMEG & Verbenaceae & $\mathrm{Z}$ & FA-FJ-BN & I & SI \\
\hline
\end{tabular}

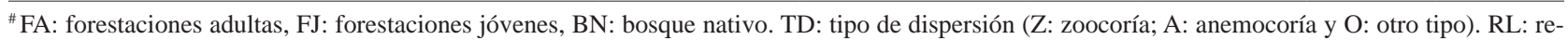
querimientos de luz (H: heliófitas; I: intermedias; E: esciófitas; -: sin datos). GE: grupos ecológicos (P: pioneras; SI: secundaria inicial; ST: secundaria tardía; C: clímax; -: sin datos). ${ }^{\dagger}$ Especie exótica.

Old plantations (FA), young plantations (FJ) and native forests (BN); Disp.: dispersion types (Z: zoochory; A: anemochory; O: other types); light requirement (H: light demander; I: intermediate; E: shade tolerant; -: no data) and ecological groups (H: light demander; I: intermediate tolerant; E: shade tolerant; -: no data). Underlined species denote exotic species. 
Grupos funcionales. Las especies más frecuentes resultaron ser las que presentaron dispersión por zoocoría (cuadro 3), representando un promedio del $72 \%$ de las especies registradas en las forestaciones adultas, $65 \%$ en las forestaciones jóvenes y $59 \%$ en el bosque nativo. Las especies con dispersión por anemocoría resultaron menos representadas, con un valor promedio de $17 \%$ en forestaciones adultas, $23 \%$ en forestaciones jóvenes y 29 \% en el bosque nativo. Las especies con otro tipo de dispersión, como por ejemplo: dispersión balística o por gravedad, presentaron valores mayores a los correspondientes para especies de dispersión anemocórica, en un intervalo de $19 \%$ a $25 \%$.

La distribución de las especies en un diagrama de ordenamiento permite diferenciar grupos de acuerdo a su forma de dispersión y tolerancia a la sombra sobre el eje $1(r=0,86 ; P<0,05)$ (figura 3). Las especies dispersadas por viento y demandantes de luz se encuentran sobre los valores negativos del eje 1 (grupo a), influenciadas por la distancia al bosque nativo y valores bajos de área basal. En el centro se ubica el grupo de las especies más frecuentes (grupo d) y en los valores positivos las especies dispersadas por animales y de mayor resistencia a las condiciones de sombra. Dentro de este grupo es factible distinguir dos grupos de especies. Las especies relacionadas a las fores- taciones provenientes de bosque nativo como uso anterior del suelo $(r=0,91 ; P<0,05)$ se encuentran sobre los valores positivos del eje 2 (grupo b) y las especies características de forestaciones provenientes de uso agrícola, sobre los valores negativos del mismo eje (figura 3 , grupo c).

Variables asociadas a la diversidad. El análisis de correspondencia canónica demostró que el 35,6 \% de la variación total de las especies en las forestaciones puede ser explicada por las variables ambientales estudiadas, resultando significativa de acuerdo a la prueba de permutación de Montecarlo ( $\mathrm{F}=18,48 ; P<0,002)$ (figura 4). Las variables que resultaron significativas para explicar la distribución de las especies en los distintos sitios fueron, de acuerdo a su importancia: uso anterior, área basal, y distancia al bosque nativo en el año 1989. La varianza explicada por los dos primeros ejes alcanzó el 77,8 \%, con valores de 39,3 \% y $38,5 \%$ para los ejes 1 y 2 , respectivamente, mientras que 22,2 \% fue representado por el eje 3.

El área basal se correlacionó con el eje $1(\mathrm{r}=0,92$; $P<0,05$ ), mientras que el uso anterior resultó fuertemente correlacionado al eje $2(\mathrm{r}=0,95 ; P<0,05)$ y la distancia al bosque nativo se relacionó al eje $3(\mathrm{r}=0,92 ; P<0,05)$. La distancia al bosque nativo resultó con valores de corre-

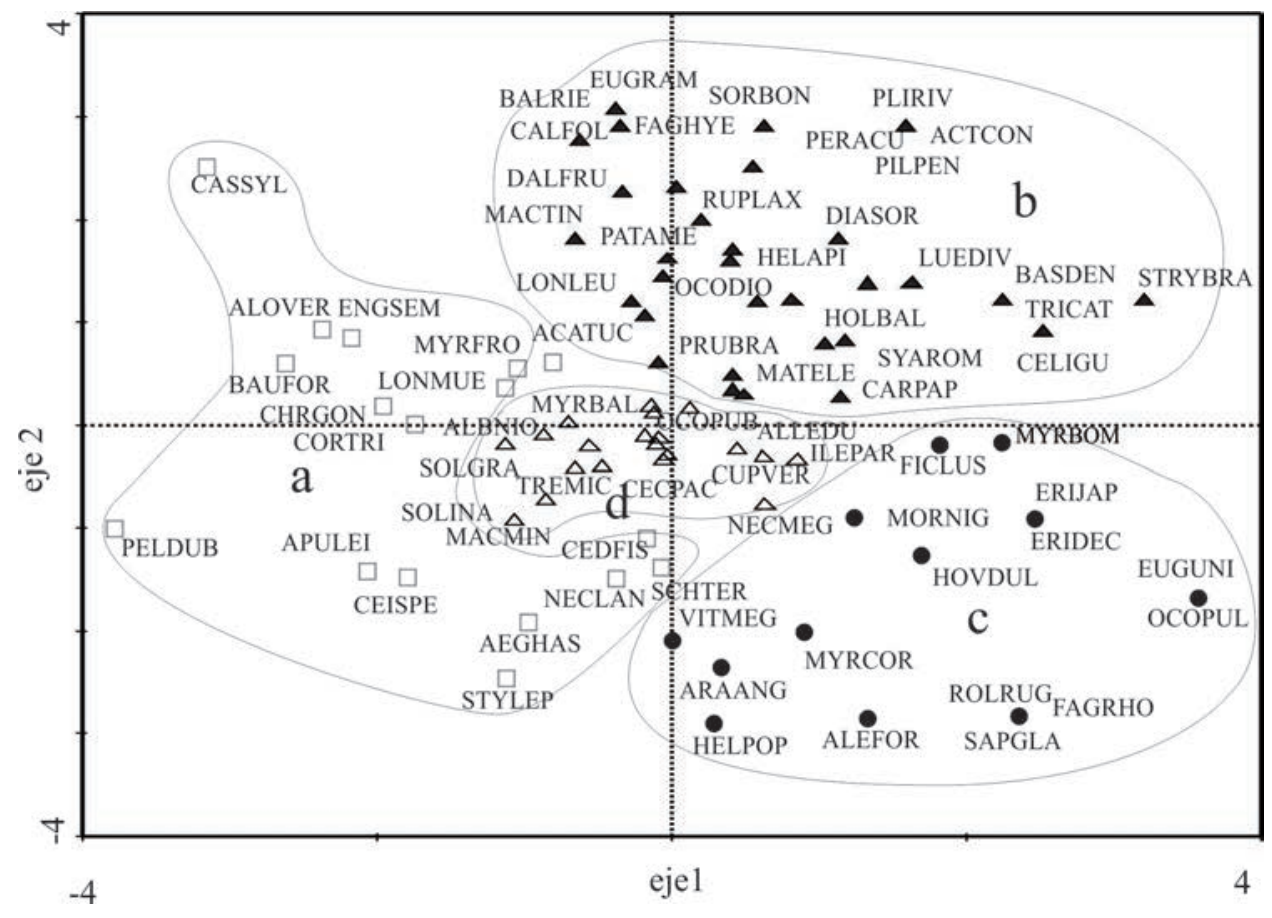

Figura 3. Diagrama de ordenamiento del análisis de correspondencia canónica para los datos de especies. Grupo a: especies pioneras/ dispersadas por viento; grupo b: especies dispersadas por animales/tolerantes a la sombra asociadas a bosque nativo; grupo c especies dispersadas por animales/tolerantes a la sombra asociadas a ambientes agrícolas; grupo d: especies más frecuentes inventariadas en la mayoría de los sitios. Los códigos representan los nombres abreviados de las especies (cuadro 3).

Ordination plot from canonical correspondence analysis of the species/environment data in the study sites. Group a: pioneer species/wind dispersed; group b: shade tolerant species/animal dispersed related to the native forest; group c: shade tolerant species/animal dispersed related to the agricultural environments; group d: frequent species present in most plots. Codes represent the scientific names of species (see table 3). 


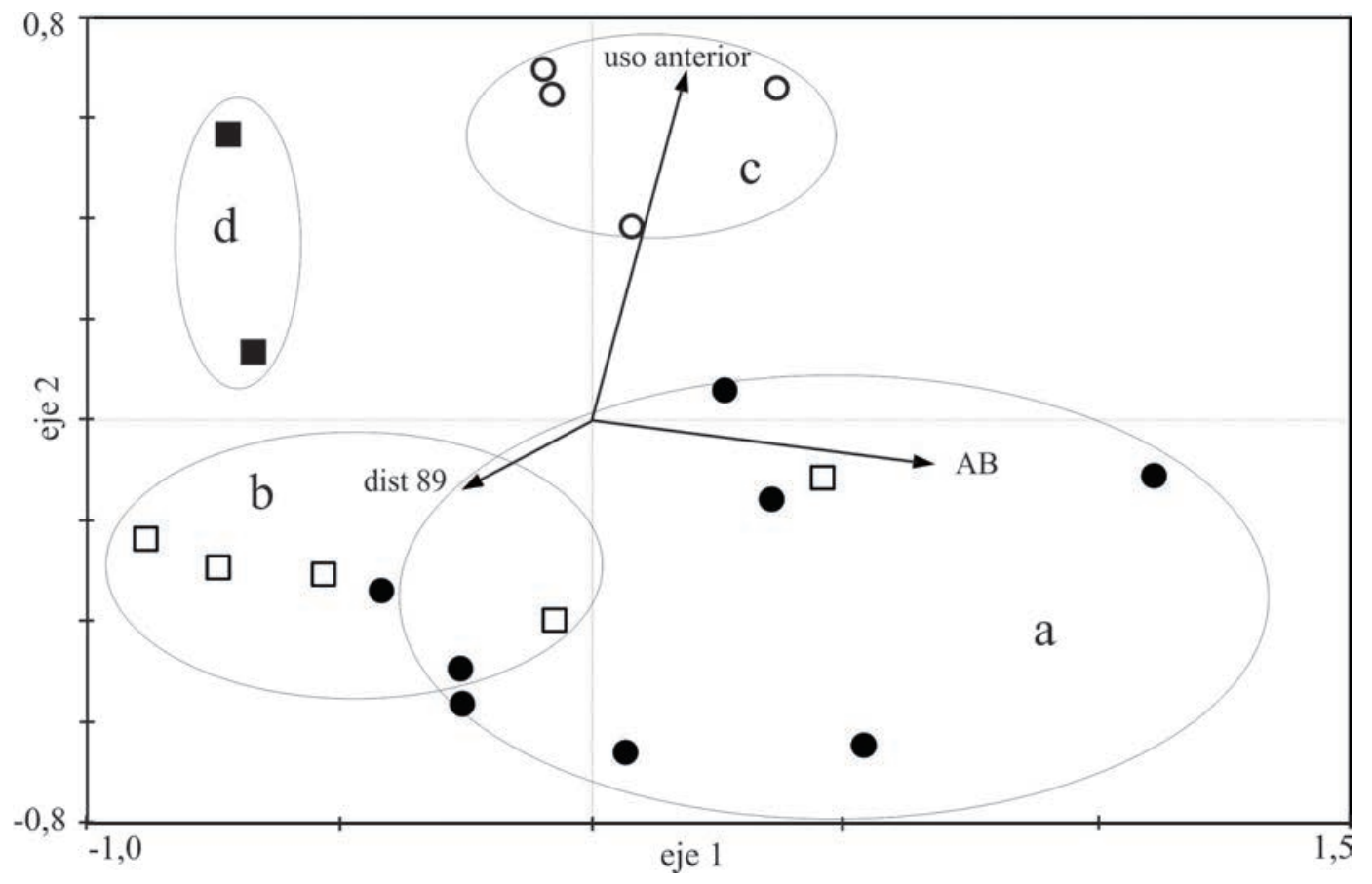

Figura 4. Diagrama de ordenamiento del análisis de correspondencia canónica de variables ambientales para los sitios estudiados. Círculos negros: forestaciones adultas; cuadrados blancos: forestaciones jóvenes. AB: área basal; Dist89: distancia al bosque nativo en el año 1989; uso anterior: uso anterior del suelo previo a la instalación de las forestaciones. Grupo a: forestaciones adultas provenientes de uso agrícola; grupo b: plantaciones jóvenes provenientes de uso agrícola; grupo c: forestaciones adultas provenientes de bosque nativo; grupo d: forestaciones jóvenes provenientes de bosque nativo.

Ordination plot from canonical correspondence analysis of the plot/environment data in the study sites. Black circles: old plantations; white squares: young plantations. AB: basal area; Dist 89: distance to the native forest at the year of planting (1989); uso anterior (previous land use): land use prior to the plantations. Group a: old plantations originated from agricultural use; group b: young plantations originated from agricultural use; group c: old plantations originated from native forest; group d: young plantations originated from native forest.

lación inferiores sobre los dos primeros ejes respecto a las otras variables $(\mathrm{r}=-0,35 ; P<0,05 \mathrm{y} \mathrm{r}=-0,19 ; P<0,05)$. En la figura 4 se separan los sitios que provienen de bosque nativo y fueron transformados a forestaciones ubicados sobre los valores positivos del eje 2 (grupos c y d) siendo posible discriminar forestaciones maduras (grupo c) y jóvenes (grupo d). Los sitios provenientes de agricultura se agruparon sobre los valores negativos del mismo eje (grupos a y b). Por otra parte, los sitios de mayor área basal, coincidentes con los sitios de más edad se distribuyeron principalmente sobre los cuadrantes positivos del eje 1 (grupos a y c) y los sitios con menor área basal se encontraron sobre los cuadrantes negativos del mismo eje (grupos b y d).

\section{DISCUSIÓN}

Los resultados presentados permiten precisar algunas de las variables que influyen sobre la composición y riqueza del sotobosque en plantaciones de Pinus taeda. Primeramente, es posible aseverar que las forestaciones permiten el desarrollo de un gran número de especies nativas en el sotobosque, aunque en cantidades significativamente menores al bosque nativo, en concordancia con diversos autores (Parrotta et al. 1997a), con trabajos realizados en la región (Andrae et al. 2004) y en países vecinos en forestaciones de otras especies del género (Gómez et al. 2009). Sin embargo, el número de especies resultó menor al informado por Andrae et al. (2004) en forestaciones de Pinus taeda en Brasil, posiblemente debido a una mayor riqueza de especies nativas en la zona de estudio de dicho trabajo respecto a los bosques misioneros.

Si bien la riqueza de leñosas en las forestaciones resultó significativamente menor que la encontrada o esperada en los bosques nativos, muchas de las especies presentes representan a aquellas que ocupan el dosel superior y, por lo tanto, es posible que en los sitios se puedan desarrollar ensambles de especies similares a las encontrados en bosques nativos (Holz et al. 2009). Esta situación permitiría que las plantaciones constituyan un elemento que facilite la conectividad del paisaje productivo de la región y constituyan una herramienta para la restauración de áreas deforestadas (Lamb 1998). Aspectos relacionados al diseño espacial de las forestaciones con los remanentes nativos como así también, aspectos de manejo de las plantaciones son de suma importancia al momento de evaluar el rol que pueden jugar las forestaciones de esta especie en la 
conectividad del paisaje y en la conservación de la biodiversidad en estos ambientes productivos (Lindenmayer y Hobbs 2004).

El aumento de la riqueza de especies en relación al desarrollo del área basal o edad de las plantaciones estaría relacionado a los cambios micro ambientales (radiación, temperatura, humedad, estructura del suelo) favorables para la regeneración y los cambios estructurales que generarían ambientes aptos para dispersores promoviendo la llegada de propágulos a medida que aumenta la edad de la forestación (Lugo 1997).

Por otra parte, la intensidad o tipo de uso anterior como variable condicionante de la composición de especies también fue observado por diferentes autores (Gachet et al. 2007, Holz et al. 2009). La implantación sobre sitios provenientes de bosques nativos o de forestaciones permitiría la existencia de un banco de propágulos y semillas que asegurarían la presencia de las especies nativas en el sotobosque, mientras que en los sitios provenientes de agricultura la eliminación de propágulos y la pérdida del banco de semillas generaría sotobosques menos diversos (Gachet et al. 2007). Por lo tanto, el manejo de la vegetación del sotobosque luego de la cosecha y durante los primeros años de instalación de las forestaciones podría tener un importante efecto en los ciclos siguientes, generando sotobosques más pobres por la generación de "efectos a largo plazo" originados por la aplicación de herbicidas y el manejo de la plantación. Estos efectos disminuirían la posibilidad de que estas forestaciones presenten cualidades diferenciales que permitan su utilización por parte de diferentes especies y con ello mejorar su rol en la conectividad del paisaje (Lindenmayer y Hobbs 2004)

La influencia de la distancia al bosque nativo en el momento de la implantación de las forestaciones indica cambios en la composición de especies en relación a la distancia debido a la limitación en la dispersión de semillas, particularmente durante los primeros años de la plantación Parrotta (1997b). Las especies dispersadas por viento serían las más influenciadas por la distancia a las fuentes de semillas (Clark et al. 2005). Esta limitación en la dispersión coincide con la influencia de la distancia al momento de plantación sobre estas especies y con la mayor proporción de especies dispersadas por animales encontradas para las plantaciones estudiadas. Sin embargo, resulta difícil discriminar la influencia de la distancia al momento de plantación como variable del paisaje sobre la riqueza de especies. La existencia semilleros aislados dentro de la forestación o en el borde del camino, que podrían haber aportado sus semillas en las forestaciones cercanas, junto a otros factores como el banco de semillas y los rebrotes como mecanismos de persistencia, podrían disminuir el peso de esta variable.

La presencia mayoritaria de especies dispersadas principalmente por aves indicaría que las forestaciones podrían representar un hábitat empleado por la fauna autóctona. Aunque se ha informado que la diversidad de aves en forestaciones es menor que la existente en formaciones nativas, principalmente representada por especies de aves generalistas (Zurita et al. 2006), los valores de riqueza para diferentes usos de la tierra indican a las forestaciones como la actividad antrópica que permite la existencia de un mayor número de especies respecto a usos tales como ganadería y cultivos anuales (Zurita y Bellocq 2010). En este sentido, forestaciones que sean manejadas a nivel de paisaje y rodal, con técnicas que permitan mayores niveles de diversidad podrían considerarse como hábitats o elementos permeables de la matriz del paisaje (Lindenmayer y Hobbs 2004, Tomasevic y Estades 2008) disminuyendo los efectos de la pérdida de hábitat y de conectividad del paisaje por cambios de uso de suelo en la provincia de Misiones. Aspectos como el arreglo espacial, el tamaño del rodal, la presencia de árboles legado y el raleo de las forestaciones junto con la presencia de remanentes de vegetación nativa deben ser considerados para que las forestaciones puedan representar un hábitat adecuado para muchas especies y asegurar la conectividad del paisaje (Lamb 1998, Lindenmayer y Hobbs 2004). El valor de las forestaciones para la fauna está íntimamente relacionado al desarrollo del sotobosque y, por lo tanto, en forestaciones con un sotobosque desarrollado es posible encontrar un mayor número de especies animales (Hobbs et al. 2003). Las actividades de raleo permiten la regeneración, desarrollo y fructificación de las especies vegetales del sotobosque generando un ambiente más atractivo para la fauna (Rishworth et al. 1995). La presencia de parches de vegetación nativa relacionados a las forestaciones permite que un mayor número de especies animales utilicen las forestaciones adyacentes (Lindenmayer y Hobbs 2004). La existencia de árboles legado es particularmente importante para las aves que pueden usar estos individuos para sus movimientos, nidificar, alimentarse y como posaderos, contribuyendo además a la dispersión de semillas dentro de las forestaciones (Lindenmayer y Hobbs 2004).

La influencia de la edad de las forestaciones sobre la diversidad de leñosas refuerza la importancia de generar forestaciones que combinen diferentes edades y que permitan mantener mejores niveles de diversidad a nivel de paisaje por sobre las forestaciones realizadas en grandes bloques coetáneos (Hartley 2002, Lindenmayer y Hobbs 2004). En función de las relaciones encontradas en este estudio entre la riqueza de especies leñosas nativas en las forestaciones y variables del rodal, como la edad o área basal y la distancia al bosque nativo durante los primeros años de la forestación, es posible reforzar la idea de que al momento de la planificación deben considerarse las características de las plantaciones a escala de sitio o rodal y la configuración del paisaje circundante. De esta manera seria posible lograr mayores niveles de diversidad en los sistemas productivos, generando gradientes ambientales que permitan la coexistencia de diferentes organismos (Lindenmayer y Hobbs 2004). A pesar de contener una menor cantidad de especies que el bosque nativo, las forestaciones 
que reemplazan a actividades agrícolas o ganaderas pueden considerarse como catalizadoras de la recuperación de los sitios y es por ello que podrían considerarse bajo esquemas de recuperación de áreas degradadas (Lugo 1997, Parrotta et al. 1997a). Por otra parte, la presencia de especies nativas de valor comercial como Cedrela fissilis, Cabralea canjerana, Patagonula americana L., Peltophorum dubium, entre otras, permite vislumbrar la posibilidad de realizar plantaciones multiespecíficas con un estrato superior de coníferas y un estrato conformado por nativas para un segundo ciclo forestal.

\section{CONCLUSIONES}

Las forestaciones de Pinus taeda pueden generar un ambiente adecuado para la instalación espontánea de especies arbóreas nativas. La edad de la forestación y el tipo de uso de suelo que antecede a la implantación son las variables que más influyen sobre la composición. Por lo tanto, estas variables deben considerarse al momento de planificar el uso del suelo si se pretende mantener la resiliencia del sistema y generar un ambiente productivo más diverso. Sin embargo, es necesario explorar aspectos como el rol del banco de semillas, el aporte proveniente de la lluvia de semillas y de rebrotes para disponer de conocimientos que permitan generar herramientas más completas para el manejo a nivel de rodal y a nivel de paisaje.

\section{AGRADECIMIENTOS}

A las empresas PINDO S.A., representada por el ingeniero forestal Hugo Reis, y TAEDA S.A., representada por el ingeniero agrónomo Jorge Ranger, por permitir la realización de los muestreos en propiedades de la empresa. Al técnico agrónomo Rubén Bischoff por el apoyo brindado y al Dr. Héctor Keller por su colaboración en la identificación de las especies. A Verónica Rusch, María Victoria Lantschner y Marcelo Arturi por sus valiosos comentarios y sugerencias. A los revisores por sus comentarios y correcciones. El presente trabajo fue financiado con fondos INTA-PNFOR2214.

\section{REFERENCIAS}

Andrae FH, R Palumbo, J Cardoso Marchiori, MA Durlo. 2004. O sub-bosque de reflorestamentos de Pinus em sítios degradados da região da floresta estacional decidual do Rio Grande do Sul. Ciencia Florestal 15: 43-63.

Brockerhoff EG, H Jactel, JA Parrotta, ChP Quine, J Sayer. 2008. Plantation forests and biodiversity: oxymoron or opportunity? Biodiversity Conservation. 17: 925-951.

Cabrera AL. 1976. Regiones Fitogeográficas Argentinas. Enciclopedia Argentina de Agricultura y Jardinería. Capital Federal. Buenos Aires. Argentina. Editorial ACME. 85 p.

Carvalho PER. 2003. Espécies Arbóreas Brasileiras. Brasília. Brasil. Empresa Brasileira de Pesquisa Agropecuária (EMBRAPA). EMBRAPA Florestas. 1039 p.
Clark CJ, JR Poulsen, Bolker BM, EF Connor, VT Parker. 2005. Comparative seed shadows of bird-, monkey-,and winddispersed trees. Ecology 86: 2684-2694.

Clarke KR. 1993. Non-parametric multivariate analyses of changes in community structure. Australian Journal of Ecology 18: $117-143$.

Deconchat M, EG Brockerhoff, L Barbaro. 2009. Effects of surrounding landscape composition on the conservation value of native and exotic habitats for native forest birds. Forest Ecology and Management 258: 196-204.

Di Bitetti MS, G Placci, LA Dietz. 2003. Una visión de biodiversidad para la eco región del bosque Atlántico del Alto Paraná: Diseño de un Paisaje para la Conservación de la Biodiversidad y prioridades para las acciones de conservación. Washington, D.C., USA. World Wildlife Fund. 156 p.

Gachet S, A Leduc, Y Bergeron, T Nguyen-Xuan, F Tremblay. 2007. Understory vegetation of boreal tree plantations: Differences in relation to previous land use and natural forests. Forest Ecology and Management 242: 49-57.

Gomez P, S Hahn, J San Martin. 2009. Estructura y composición florística de un matorral bajo plantaciones de Pinus radiata D. Don en Chile central. Gayana Botánica 66: 256-268.

Hammer Ø, DAT Harper, PD Ryan. 2001. PAST: Palaeontological statistics software package for education and data analysis. Palaentologica Electronica 4:9 p. Disponible en: http:// palaeo-electronica.org/2001_1/past/issue1_01.htm

Hartley MJ. 2002. Rationale methods for conserving biodiversity in plantation forests. Forest Ecology and Management 155: 81-95.

Hobbs RJ, P Catling, JC Wombey, M Clayton, L Atkins, A Reid. 2003. Faunal use of Bluegum (Eucalyptus globulus) plantations in southwestern Australia. Agroforestry Systems 58: 195-212.

Holz S, G Placci, RD Quintana. 2009. Effects of history of use on secondary forest regeneration in the Upper Parana Atlantic Forest (Misiones, Argentina). Forest Ecology and Management 258: 1629-1642.

Lamb D.1998. Large-scale Ecological Restoration of Degraded Tropical Forest Lands: The Potential Role of Timber Plantations. Restoration Ecology 6: 271-279.

Lindenmayer DB, RJ Hobbs. 2004. Fauna conservation in australian plantation forests: a review. Biological Conservation 119: $151-168$.

Lugo AE. 1992. Comparison of tropical tree plantations with secondary forests of similar age. Ecological Monographs 62: 1-41.

Lugo AE. 1997. The apparent paradox of re-establishing species richness on degraded lands with the tree monocultures. Forest Ecology and Management 99: 9-19.

McGarigal K, SA Cushman, MC Neel, E Ene. 2002. FRAGSTATS: Spatial pattern analysis program for categorical maps. Computer software program. Amherst, USA. University of Massachusetts. Disponible en: www.umass.edu/ landeco/research/fragstats/fragstats.html

Olson DM, E Dinerstein. 2002. The global 200: Priority ecoregions for global conservation. Annals of the Missouri Botanical Garden 89: 129-224.

Parrotta JA, JW Turnbull, N Jones. 1997a. Catalyzing native forest regeneration on degraded tropical land. Forest Ecology and Management 99: 1-7.

Parrotta JA, OH Knowles, JM Wunderle Jr. 1997b. Development 
of floristic diversity in 10-year-old restoration forests on a bauxited mined site in Amazonia. Forest Ecology and Management 99: 21-42.

Rishworth C, J McIIroy, MT Tanton. 1995. Factors affecting population densities of the Common Wombat Vombatus ursinus in plantations of Pinus radiata. Forest Ecology and Management 76: 11-19.

SAGPyA. 2001. Sector Forestal. Dirección de Forestación. Buenos Aires, Argentina. Secretaría de Agricultura, Ganadería, Pesca y Alimentación. 85 p.

Stohlgren TJ. 2007. Measuring Plant Diversity. New York. USA. Oxford University Press. 390 p.

Ter Braak CJF, P Šmilauer. 1998. Reference manual and user's guide to canoco for windows: software for canonical community ordination (version 4). New York. USA. Microcomputer Power. $352 \mathrm{p}$.
Tomasevic JA, CF Estades. 2008. Effects of the structure of pine plantations on their "softness" as barriers of ground-dwelling forest birds in south-central Chile. Forest Ecology and Management 255: 810-816.

Turner MG, RH Gardner, RV O’Neill. 2001. Landscape Ecology in theory and practice. Pattern and process. New York. USA. Springer Verlag. 401 p.

Zurita GA, N Rey, DM Varela, M Villagra, MI Bellocq. 2006. Conversion of the Atlantic Forest into native and exotic tree plantations: Effects on bird communities from the local and regional perspectives. Forest Ecology and Management 235: 164-173.

Zurita GA, MI Bellocq. 2010. Spatial patterns of bird community similarity: bird responses to landscape composition and configuration in the Atlantic Forest. Landscape Ecology 25: 147-158.

Recibido: 25.04.13

Aceptado: 07.10.13 